\title{
Editorial: Induced Pluripotent Stem Cell-Based Disease Modeling and Drug Discovery: Can We Recapitulate Cardiovascular Disease on a Culture Dish?
}

\begin{abstract}
Shinsuke Yuasa ${ }^{1 *}$, Masayuki Yazawa ${ }^{2,3,4}$ and Jong-Kook Lee ${ }^{5 *}$
${ }^{1}$ Department of Cardiology, Keio University School of Medicine, Tokyo, Japan, ${ }^{2}$ Department of Rehabilitation and Regenerative Medicine, Vagelos College of Physicians and Surgeons, Columbia University, New York City, NY, United States, ${ }^{3}$ Department of Molecular Pharmacology and Therapeutics, Vagelos College of Physicians and Surgeons, Columbia University, New York City, NY, United States, ${ }^{4}$ Columbia Stem Cell Initiative, Vagelos College of Physicians and Surgeons, Columbia University, New York City, NY, United States, ${ }^{5}$ Department of Cardiovascular Regenerative Medicine, Osaka University Graduate School of Medicine, Suita, Japan
\end{abstract}

Keywords: iPS cell, cardiomyocyte, endothelial cell, disease modeling, drug discovery

Editorial on the Research Topic

Induced Pluripotent Stem Cell-Based Disease Modeling and Drug Discovery: Can We Recapitulate Cardiovascular Disease on a Culture Dish?

Since induced pluripotent stem (iPS) cells were first generated in 2006 (Takahashi and Yamanaka, 2006), human iPS cell culture and differentiation into various cell types have been widely established as research platforms for elucidating disease mechanisms and for drug discovery research, and promising cell sources for regenerative medicine (Tanaka et al., 2015). Human iPS cells can be generated from patient's somatic cells, such as skin fibroblasts or blood cells, and the approach using iPS cells offers an ethical advantage over embryonic stem cells (Seki et al., 2010; Seki et al., 2012). There have been a variety of established methods to differentiate many cell types from human iPS cells. In terms of cardiovascular disease, recent accumulating progress has enabled human iPS cells to be efficiently differentiated and purified into subtypes of cardiomyocytes, such as ventricular-, atrialand nodal-like myocytes, as well as smooth muscle cells and endothelial cells. Based on these advances, disease-specific iPS cells can be generated from patients with intractable cardiovascular diseases, such as inherited cardiomyopathies and arrhythmic disorders. There are substantial reports to elucidate the molecular and cellular mechanisms underlying disease onset and/or the pathophysiological process through various disease-specific iPS cell studies (Yazawa et al., 2011; Egashira et al., 2012; Tanaka et al., 2014; Yasutake et al., 2021). It is also conceivable that iPS cellderived cardiomyocytes can be utilized for drug screening. For robust applications using human iPS cells for research and clinics, there remains several barriers to be overcome, such as immaturity of iPS cell-derived cardiomyocytes, lack of cell-cell interaction, and absence of organ structure. Many studies still struggle against these issues, which should be solved step by step.

Although iPS cells are supposedly differentiated into any types of cells, most of the differentiated cells that are currently available show immature/fetal feature. To model adult-onset diseases, it is required to mature the differentiated cells. In terms of regeneration therapy, immature cardiomyocyte transplantation may induce arrhythmic event and reduce the efficacy of functional recovery. To mature cardiomyocytes, it is important to understand the difference between embryonic and adult cardiomyocytes. Anzai et al. identified gene sets to monitor the developmental stage in murine and human cardiomyocytes. Comparative transcriptome data analysis of mouse and human hearts in several developmental 
stages revealed the difference and similarity of two species. While some reports previously showed maturation markers, the proposed markers were either mouse specific or human specific. Importantly, identified gene sets can be markers for both mouse and human cardiomyocyte maturation. Li et al. reported their cardiomyocyte maturation method in human iPS cell-derived cardiomyocytes. Aligned fiber substrate culture induced mature-like properties including rod shape morphology, shortened action potential duration, accelerated conduction velocity, and elevated adult-type gene expression. Contractility of the heart increases as its beating rate is elevated, which is observed in human matured heart, but not in mouse heart and immature iPS cell-derived cardiomyocytes. IzumiNakaseko et al. reported that motion directional regulation by electrical pacing could induce positive force-frequency relationship in monolayers of human iPS cell-derived cardiomyocytes. These findings facilitate us to establish sophisticated methods to mature cardiomyocytes and analysis method to understand the molecular and cellular pathophysiological mechanisms underlying heart diseases.

To model human cardiovascular disease and establish regeneration therapy using iPS cell-derived cells, it is essential for us to develop new methods to differentiate iPS cells into cardiovascular cells and understand the properties of iPS cellderived cells. Although endothelial progenitor cells (EPCs) have been used for vascular regeneration therapy, it is inefficient to obtain EPCs from adult donors. Farkas et al. reported the efficient protocol to differentiate EPCs from human iPS cells. As for EPCs, immature phenotype is not disadvantageous because mature endothelial cells lack angiogenic and vasculogenic potential. However, to use iPS cellderived immature cardiomyocytes, it is critically important to understand the difference from adult cardiomyocytes. Muscular dystrophies are caused by the mutation in genes encoding the protein involved in the dystrophin-associated protein complex (DAPC). Cardiomyopathies are crucial phenotypes and could be a lethal cause. To model this disease, it is important to examine the expression and function of DAPC in iPS cell-derived cardiomyocytes. Gilbert et al. showed the robust expression of DYSTROPHIN, but the absence of several other DAPC proteins, suggesting that it is still difficult to model the disease using current immature cardiomyocytes. The type 2 ryanodine receptor (RYR2) is an essential $\mathrm{Ca}^{2+}$ release channel of sarcoplasmic reticulum (SR) in adult cardiomyocyte. Luo et al., generated RYR2-/- iPS cell line and investigated the role of RYR2 in iPS cell-derived cardiomyocytes. RYR2 is not required for cardiomyocyte differentiation but plays a role in survival and contractile function. Without RyR2, another calcium release channel, IP3R mediates $\mathrm{Ca}^{2+}$ release as a compensatory mechanism for $\mathrm{Ca}^{2+}$ handling in iPS cell-derived cardiomyocyte.

Atrial fibrillation (AF) is the most common cardiac arrhythmic disease. Loss of function and gain of function mutations in KCNA5, encoding the Kv1.5a-subunit of the ion channel carrying the atrialspecific ultrarapid delayed rectifier $\mathrm{K}^{+}$current $\left(\mathrm{I}_{\mathrm{kur}}\right)$ are reported in approximately $10 \%$ of the patients with AF. Hilderink et al. examined the effects of virtual $\mathrm{I}_{\mathrm{Kur}}$ injection in iPS cell-derived atrial-like cardiomyocyte because native $\mathrm{I}_{\mathrm{Kur}}$ density is too small to be examined. Virtual modulation study revealed that a decrease in $I_{\text {Kur, }}$ mimicking loss-of-function mutations, significantly prolonged action potential duration, but an increase in $I_{\text {Kur, }}$ mimicking gain-offunction mutations, mildly shortened that in iPS cell-derived atriallike cardiomyocyte. Long QT (LQT) syndrome is an inherited lifethreatening arrhythmogenic disease. Although recent report showed the efficacy of $\beta$-blockers on the LQT type3 (LQT3) (Wilde et al., 2016), the pharmacological mechanism remains unclear. Hirose et al. modeled the LQT3 and confirmed the efficacy of $\beta$-blocker in the model. Interestingly, $\beta$-blocker reduced the disease-causing late sodium current in the presence of guanosine diphosphate $\beta \mathrm{s}$ (GDP $\beta s$ ), an inhibitor of G proteins, suggesting that the effect of $\beta$ blocker may be independent of $\beta$-adrenergic receptor. Hypoplastic left heart syndrome (HLHS) is a severe form of congenital heart disease. While genetic variants in MYH6 are reported, the disease mechanism remains unknown. Kim et al. model HLHS by iPS cellderived cardiomyocytes and showed multiple impairment in cardiomyocyte differentiation, sarcomere organization, slower contraction, and decreased velocity phenotypes. HLHS patients sample showed sarcomere disorganization in atrial but not ventricular tissues. These results suggest that reduced contractility in atrium of HLHS patients may negatively affect hemodynamics and result in the development of a left ventricle.

Cardiovascular diseases are highly prevalent globally as a main cause of death. Several therapeutic strategies have been developed, such as medical therapy, catheter intervention, cardiac assist device, and heart transplantation. However, the therapeutics remain insufficient, and we still require innovative approaches to improve the therapeutics. To develop the therapeutics further, it is important to understand the disease pathogenesis and develop the drug discovery and optimization platforms. Various innovative analysis methods have been proposed, developed, and contributed to the cardiovascular research (Kusumoto et al., 2018; Kusumoto and Yuasa, 2019; Kusumoto et al., 2021). Therefore, the research using human iPS cells with genetic variants is still highly valuable. Continuous iPS cell study will allow us to understand the diseases and develop the innovative therapy to improve human health.

\section{AUTHOR CONTRIBUTIONS}

All authors listed have made a substantial, direct, and intellectual contribution to the work and approved it for publication.

\section{FUNDING}

This work was supported by JSPS KAKENHI (grant numbers 20H03678, 20K08193, 20K08461, 19H03622), Keio University Academic Development Funds for Individual Research, and SENSHIN Medical Research Foundation (SY). The study was partially supported by the Agency for Medical Research and Development, AMED (JP17bm0804008h0001 to J-KL), JSPS KAKENHI (Grant Number JP18H03517), and Co-Create Knowledge for Pharma Innovation with Takeda (COCKPI$\mathrm{T}^{\circledR}$ ) Funding $(\mathrm{J}-\mathrm{KL})$. This study received funding from Alchemedicine, Inc. (SY), Screen Holdings Co. Ltd (J-KL) 
and Alpha MED Scientific, Inc. (J-KL). The funders were not involved in the study design, collection, analysis, interpretation of data, the writing of this article or the decision to submit it for publication.

\section{REFERENCES}

Egashira, T., Yuasa, S., Suzuki, T., Aizawa, Y., Yamakawa, H., Matsuhashi, T., et al. (2012). Disease Characterization Using LQTS-specific Induced Pluripotent Stem Cells. Cardiovasc. Research.95 95, 419-429. doi:10.1093/cvr/cvs206

Kusumoto, D., Lachmann, M., Kunihiro, T., Yuasa, S., Kishino, Y., Kimura, M., et al. (2018). Automated Deep Learning-Based System to Identify Endothelial Cells Derived from Induced Pluripotent Stem Cells. Stem Cel Rep. 10 (10), 1687-1695. doi:10.1016/j.stemcr.2018.04.007

Kusumoto, D., Seki, T., Sawada, H., Kunitomi, A., Katsuki, T., Kimura, M., et al. (2021). Anti-senescent Drug Screening by Deep Learning-Based Morphology Senescence Scoring. Nat Commun. Jan 11 (12), 257. doi:10.1038/s41467-02020213-0

Kusumoto, D., and Yuasa, S. (2019). The Application of Convolutional Neural Network to Stem Cell Biology. Inflamm. Regen. 39 (39), 14. doi:10.1186/s41232019-0103-3

Seki, T., Yuasa, S., and Fukuda, K. (2012). Generation of Induced Pluripotent Stem Cells from a Small Amount of Human Peripheral Blood Using a Combination of Activated T Cells and Sendai Virus. Nat. Protoc. 7 (7), 718-728. doi:10.1038/nprot.2012.015

Seki, T., Yuasa, S., Oda, M., Egashira, T., Yae, K., Kusumoto, D., et al. (2010). Generation of Induced Pluripotent Stem Cells from Human Terminally Differentiated Circulating T Cells. Cell Stem Cell. 7 (7), 11-14. doi:10.1016/ j.stem.2010.06.003

Takahashi, K., and Yamanaka, S. (2006). Induction of Pluripotent Stem Cells from Mouse Embryonic and Adult Fibroblast Cultures by Defined Factors. Cell. 126 (126), 663-676. doi:10.1016/j.cell.2006.07.024

Tanaka, A., Yuasa, S., Mearini, G., Egashira, T., Seki, T., Kodaira, M., et al. (2014). Endothelin-1 Induces Myofibrillar Disarray and Contractile Vector Variability in Hypertrophic Cardiomyopathy-Induced Pluripotent Stem Cell-Derived Cardiomyocytes. J. Am. Heart Assoc. 3, e001263. doi:10.1161/ JAHA.114.001263

\section{ACKNOWLEDGMENTS}

We would like to thank all the members in laboratory for helpful discussion.

Tanaka, A., Yuasa, S., Node, K., and Fukuda, K. (2015). Cardiovascular Disease Modeling Using Patient-specific Induced Pluripotent Stem Cells. Int. J. Mol. Sci. 16, 18894-18922. doi:10.3390/ijms160818894

Wilde, A. A. M., Moss, A. J., Kaufman, E. S., Shimizu, W., Peterson, D. R., Benhorin, J., et al. (2016). Clinical Aspects of Type 3 Long-QT Syndrome. Circulation 134, 872-882. doi:10.1161/circulationaha.116.021823

Yasutake, H., Lee, J. K., Hashimoto, A., Masuyama, K., Li, J., Kuramoto, Y., et al. (2021). Decreased YAP Activity Reduces Proliferative Ability in Human Induced Pluripotent Stem Cell of Duchenne Muscular Dystrophy Derived Cardiomyocytes. Sci. Rep. 11 (11), 10351. doi:10.1038/s41598-021-89603-8

Yazawa, M., Hsueh, B., Jia, X., Pasca, A. M., Bernstein, J. A., Hallmayer, J., et al. (2011). Using Induced Pluripotent Stem Cells to Investigate Cardiac Phenotypes in Timothy Syndrome. Nature 471 (471), 230-234. doi:10.1038/ nature09855

Conflict of Interest: The authors declare that the research was conducted in the absence of any commercial or financial relationships that could be construed as a potential conflict of interest.

Publisher's Note: All claims expressed in this article are solely those of the authors and do not necessarily represent those of their affiliated organizations, or those of the publisher, the editors and the reviewers. Any product that may be evaluated in this article, or claim that may be made by its manufacturer, is not guaranteed or endorsed by the publisher.

Copyright (c) 2022 Yuasa, Yazawa and Lee. This is an open-access article distributed under the terms of the Creative Commons Attribution License (CC BY). The use, distribution or reproduction in other forums is permitted, provided the original author(s) and the copyright owner(s) are credited and that the original publication in this journal is cited, in accordance with accepted academic practice. No use, distribution or reproduction is permitted which does not comply with these terms. 